\title{
TAME MEASURES ON CERTAIN COMPACT SETS
}

\author{
HSUAN-PEI LEE
}

AbSTRaCt. A finite complex Borel measure $\mu$ on a compact subset $X \subset \mathbf{C}^{n}$ is called tame if there exist finite measures $\sigma_{1}, \ldots, \sigma_{n}$ on $X$ with

$$
\int_{X} \phi d \mu=\int_{X} \sum_{1}^{n} \frac{\partial \phi}{\partial \bar{z}_{j}} d \sigma_{j}
$$

for every $\phi \in C_{0}^{\infty}\left(C^{n}\right)$. We define $X_{T}=\left\{\left(z_{1}, z_{2}\right):\left|z_{1}\right|^{2}+\left|z_{2}\right|^{2}=1\right.$ and $\left.z_{1} \in T\right\}$, where $T$ is a compact subset of $\left\{\left|z_{1}\right|<1\right\}$ in $\mathbf{C}^{1}$. It is shown in this paper that tame measures form a weak-* dense subset of $R\left(X_{T}\right)^{\perp}$. It follows then, with the help of a theorem by Weinstock, that $R\left(X_{T}\right)$ is a local algebra.

Let $X$ be a compact set in $\mathbf{C}^{n}$. $C(X)$ is the algebra of all continuous functions on $X$. $R_{0}(X)$ is the algebra of all rational functions $P / Q$ on $\mathbf{C}^{n}$ with $P, Q$ polynomials and $Q \neq 0$ on $X . R(X)$ is the uniform closure of $R_{0}(X)$ in $C(X)$.

It is a well-known consequence of Cauchy-Green formula that if $\mu$ is a complex Borel measure with compact support $X \subset \mathbf{C}^{\mathbf{l}}$, then

$$
\int \phi d \mu=-\frac{1}{2 \pi i} \int \frac{\partial \phi}{\partial \bar{z}}\left(\int \frac{1}{\zeta-z} d \mu(\zeta)\right) d z \wedge d \bar{z}
$$

holds for every $\phi \in C_{0}^{\infty}(\mathbf{C})$. It follows that $\mu$ is an orthogonal measure for $R(X)$ iff

$$
\hat{\mu}=\int \frac{1}{\zeta-z} d \mu(\zeta)
$$

is supported on $X$, or, equivalently, the measure $\hat{\mu}(z) d z \wedge d \bar{z}$ is supported on $X$. This gives a description of orthogonal measures for $R(X)$ where $X \subset \mathrm{C}$. While no general description for measures on $X \subset \mathrm{C}^{n}, n>1$, orthogonal to $R(X)$ is available, we introduce the following definition.

Definition. Let $X$ be a compact set in $\mathbf{C}^{n}$. A finite complex Borel measure is tame if there exist finite measures $\sigma_{1}, \ldots, \sigma_{n}$ on $X$ with

$$
\int_{X} \phi d \mu=\int_{X} \sum_{1}^{n} \frac{\partial \phi}{\partial \bar{z}_{j}} d \sigma_{j} \quad \text { for every } \phi \in C_{0}^{\infty}\left(\mathbf{C}^{n}\right) .
$$

(1) and (2) now imply that for $X \subset \mathbf{C}$ a measure $\mu$ on $X$ is orthogonal to $R(X)$ iff $\mu$ is tame.

Let $X \subset \mathbf{C}^{n}$ be fixed. Suppose that a tame measure $\mu$ exists on $X$ with $\mu \neq 0$. If $\phi \in R_{0}(X)$ then $\partial \phi / \partial \bar{z}_{j} \equiv 0$ on $X$ for all $j$. So by $(*) \int_{X} \phi d \mu=0$. It follows that $\mu \perp R(X)$ and hence $R(X) \neq C(X)$. Thus the existence of tame measures imply that $R(X) \neq C(X)$.

Received by the editors September 4, 1979.

AMS (MOS) subject classifications (1970). Primary 46J10.

Key words and phrases. Tame measure, weak-* dense, local algebra. 
In this paper, we restrict ourselves to subsets of $\partial B=\left\{\left(z_{1}, z_{2}\right):\left|z_{1}\right|^{2}+\left|z_{2}\right|^{2}=1\right\}$ which have the form $X_{T}=\left\{\left(z_{1}, z_{2}\right) \in \partial B: z_{1} \in T\right\}$ where $T$ is a compact subset of $\left\{\left|z_{1}\right|<1\right\}$ in $\mathbf{C}$. We study orthogonal measures for $R\left(X_{T}\right)$ and problems related to these measures.

Basener [1] has constructed a compact subset $\tilde{X}_{T}$ (which has the above stated form) of $\partial B$ such that $\tilde{X}_{T}$ is rationally convex, yet $R\left(\tilde{X}_{T}\right) \neq C\left(\tilde{X}_{T}\right)$. In the following, we will construct an ample family of tame measures for $R\left(X_{T}\right)$, provided that $R(T) \neq C(T)$. In fact, they form a weak-* dense set of $R\left(X_{T}\right)^{\perp}$. This gives an alternative explanation why $R\left(\tilde{X}_{T}\right) \neq C\left(\tilde{X}_{T}\right)$. Moreover, the weak-* density along with a theorem of Weinstock [2] lead to the conclusion that $R\left(X_{T}\right)$ is a local algebra. I.e., if $\left\{U_{\alpha}\right\}$ is a finite open covering of $X_{T}$ and if $f \in C\left(X_{T}\right)$ is such that $\left.f\right|_{X_{T} \cap \bar{U}_{\alpha}}$ is in $R\left(X_{T} \cap \bar{U}_{\alpha}\right)$, for all $\alpha$, then $f \in R\left(X_{T}\right)$. The main results may be stated as follows.

TheOREM 1. Assume that $R(T) \neq C(T)$. Then the set of tame measures on $X_{T}$ is weak-* dense in the set of all orthogonal measures to $R\left(X_{T}\right)$ on $X_{T}$.

THEOREM 2. Let $\phi$ be a smooth function with $\partial \phi / \partial \bar{z}_{i} \equiv 0$ on $X_{T}, i=1,2$. Then $\phi \in R\left(X_{T}\right)$.

TheOREM 3. $R\left(X_{T}\right)$ is a local algebra.

Notations.

$$
\begin{aligned}
B & =\left\{\left(z_{1}, z_{2}\right):\left|z_{1}\right|^{2}+\left|z_{2}\right|^{2}<1\right\}, \\
\partial B & =\left\{\left(z_{1}, z_{2}\right):\left|z_{1}\right|^{2}+\left|z_{2}\right|^{2}=1\right\}, \\
\Delta & =\left\{z_{1} \in \mathbf{C}:\left|z_{1}\right|<1\right\}, \\
X_{T} & =\left\{\left(z_{1}, z_{2}\right) \in \partial B, z_{1} \in T\right\} \quad \text { where } T \text { is a compact subset of } \Delta, \\
\Gamma_{z_{1}} & =\left\{\left(z_{1},\left(1-z_{1} \bar{z}_{1}\right)^{1 / 2} e^{i \theta}\right):-\pi \leqslant \theta<\pi\right\} .
\end{aligned}
$$

Let $\phi$ be any smooth function in a neighborhood of $X_{T}$. Let $\tilde{\phi}$ denote the composite $\phi \circ p$ where $p$ is the map from $\left\{\left|z_{1}\right| \leqslant 1\right\} \times[-\pi, \pi]$ to $\partial B$ defined by $p\left(z_{1}, \theta\right)=\left(z_{1},\left(1-z_{1} \bar{z}_{1}\right)^{1 / 2} e^{i \theta}\right)$. For each fixed $z_{1} \in T, \tilde{\phi}$ has the following Fourier expansion on $\Gamma_{z_{1}}$ :

$$
\phi\left(z_{1}, z_{2}\right)=\tilde{\phi}\left(z_{1}, \theta\right)=\sum_{-\infty}^{\infty} \phi_{n}\left(z_{1}\right) e^{i n \theta}, \quad z_{2}=\left(1-z_{1} \bar{z}_{1}\right)^{1 / 2} e^{i \theta}
$$

where

$$
\phi_{n}\left(z_{1}\right)=\int_{-\pi}^{\pi} \tilde{\phi}\left(z_{1}, t\right) e^{-i n t} \frac{d t}{2 \pi}
$$

is the $n$th Fourier coefficient of $\tilde{\phi}\left(z_{1}, \theta\right)$.

It is well known that

(i) $\phi_{n}\left(z_{1}\right)$ is smooth in $z_{1}$,

(ii) if $n \neq 0,\left|\phi_{n}\left(z_{1}\right)\right| \leqslant M / n^{3}$ for all $z_{1} \in T$ where $M$ is a constant depending on $\phi$. 
THEOREM 1. Assume that $R(T) \neq C(T)$. Then the set of tame measures on $X$ is weak-* dense in the set $R\left(X_{T}\right)^{\perp}$ of all measures on $X_{T}$ orthogonal to $R\left(X_{T}\right)$.

Proof. Let $\nu$ be a nonzero orthogonal measure for $R(T)$. Consider the linear functional which assigns to each $f$ in $C\left(X_{T}\right)$ the value

$$
\int_{T}\left(\frac{1}{2 \pi i} \int_{\Gamma_{z_{1}}} f\left(z_{1}, z_{2}\right) \frac{d z_{2}}{z_{2}}\right) d \nu\left(z_{1}\right) .
$$

Since $T$ is a compact subset of $\Delta$, we have $X_{T} \cap\left\{z_{2}=0\right\}=\phi$. Hence the above is well defined. It is easy to see that this linear functional is continuous, therefore it defines a measure $\mu$ on $X_{T}$, i.e.

$$
\int f d \mu=\int\left(\frac{1}{2 \pi i} \int_{\Gamma_{z_{1}}} f\left(z_{1}, z_{2}\right) \frac{d z_{2}}{z_{2}}\right) d \nu\left(z_{1}\right) \text { for all } f \in C\left(X_{T}\right) .
$$

ASSERTION. $\mu$ is tame.

Let $\phi \in C_{0}^{\infty}\left(\mathbf{C}^{2}\right)$,

$$
\begin{aligned}
\int_{X_{T}} \phi d \mu & =\int\left(\frac{1}{2 \pi i} \int_{\Gamma_{z_{1}}} \phi\left(z_{1}, z_{2}\right) \frac{d z_{2}}{z_{2}}\right) d \nu\left(z_{1}\right) \\
& =\int_{T}\left(\frac{1}{2 \pi} \int_{-\pi}^{\pi} \tilde{\phi}\left(z_{1}, \theta\right) d \theta\right) d \nu\left(z_{1}\right) \\
& =\int_{T} \phi_{0}\left(z_{1}\right) d \nu\left(z_{1}\right) \\
& =\frac{-1}{2 \pi i} \int \frac{\partial \phi_{0}}{\partial \bar{\zeta}_{1}} \hat{\nu}\left(\zeta_{1}\right) d \zeta_{1} \wedge d \bar{\zeta}_{1} \quad \text { by }(1) .
\end{aligned}
$$

Assume the following lemma which will be proved later.

LEMMA.

$$
\frac{\partial \phi_{0}}{\partial \bar{\zeta}_{1}}=\left(\frac{\partial \phi}{\partial \bar{\zeta}_{1}}-\frac{\zeta_{1}}{\zeta_{2}} \frac{\partial \phi}{\partial \bar{\zeta}_{2}}\right)_{0}
$$

the zeroth Fourier coefficient of $\partial \phi / \partial \bar{\zeta}_{1}-\left(\zeta_{1} / \zeta_{2}\right)\left(\partial \phi / \partial \bar{\zeta}_{2}\right)$.

We get that

$$
\begin{aligned}
\int_{X_{T}} \phi d \mu & =\frac{-1}{2 \pi i} \int\left(\frac{\partial \phi}{\partial \bar{\zeta}_{1}}-\frac{\zeta_{1}}{\zeta_{2}} \frac{\partial \phi}{\partial \bar{\zeta}_{2}}\right)_{0} \hat{\nu}\left(\zeta_{1}\right) d \zeta_{1} \wedge d \bar{\zeta}_{1} \\
& =\frac{-1}{2 \pi i} \int\left(\frac{1}{2 \pi i} \int_{\Gamma_{\zeta_{1}}}\left(\frac{\partial \phi}{\partial \bar{\zeta}_{1}}-\frac{\zeta_{1}}{\zeta_{2}} \frac{\partial \phi}{\partial \bar{\zeta}_{2}}\right) \frac{d \zeta_{2}}{\zeta_{2}}\right) \hat{\nu}\left(\zeta_{1}\right) d \zeta_{1} \wedge d \bar{\zeta}_{1}
\end{aligned}
$$

Let $\sigma_{1}$ be the measure on $\partial B$ such that for $f$ in $C(\partial B)$,

$$
\int f\left(z_{1}, z_{2}\right) d \sigma_{1} \equiv \int \frac{1}{4 \pi^{2}}\left(\int_{\Gamma_{z_{1}}} f\left(z_{1}, z_{2}\right) \frac{d z_{2}}{z_{2}}\right) \hat{\nu}\left(z_{1}\right) d z_{1} \wedge d \bar{z}_{1}
$$

and let $\sigma_{2}=-\left(z_{1} / z_{2}\right) \sigma_{1}$. 
Again, the above definitions are legitimate, for, $\nu \perp R(T)$ implies that $\hat{\nu}\left(z_{1}\right)=0$ outside $T$. This also shows that $\sigma_{1}, \sigma_{2}$ are supported on $X_{T}$. To sum up, we have shown that for any $\phi \in C_{0}^{\infty}\left(\mathbf{C}^{2}\right)$,

$$
\int \phi d \mu=\int \frac{\partial \phi}{\partial \bar{z}_{1}} d \sigma_{1}+\int \frac{\partial \phi}{\partial \bar{z}_{2}} d \sigma_{2}
$$

where $\sigma_{i}$ 's are supported on $X_{T}$. Hence $\mu$ is tame. $\mu$ is not a zero measure because $\int f\left(z_{1}\right) d \mu=\int f\left(z_{1}\right) d \nu$ for all $f$ in $C\left(\mathbf{C}^{1}\right)$ and $\nu$ is nonzero by hypothesis.

We note that if $\mu$ is a tame measure on $X \subset \mathbf{C}^{n}$, then $f \mu$ is also tame for smooth function $f$ with $\partial f / \partial \bar{z}_{i} \equiv 0$ on $X, i=1, \ldots, n$. In particular, if $\mu$ is as in (**), the measures $z_{2}^{m} \mu, m= \pm 1, \pm 2, \ldots$, are all tame. Let $S=\left\{z_{2}^{m} \mu\right.$ : there is a nonzero orthogonal measure $\nu$ for $R(T)$ such that $\mu$ is defined by $(* *), m=$ $0, \pm 1, \pm 2, \ldots\}$.

We will show that

"If $f$ in $C\left(X_{T}\right)$ is such that $f$ is annihilated by all elements in $S$, then $f$ is in $R\left(X_{T}\right)$."

Let $\Sigma_{-\infty}^{\infty} f_{n}\left(z_{1}\right) e^{i n \theta}$ be the "formal" Fourier expansion for $\tilde{f}\left(z_{1}, \theta\right)=f \circ p\left(z_{1}, \theta\right)=$ $f\left(z_{1}, z_{2}\right)$ on $\Gamma_{z_{1}}$. Let $\sigma_{j}\left(z_{1}, z_{2}\right)=\tilde{\sigma}_{j}\left(z_{1}, \theta\right)$ be the $j$ th Cesaro mean for $\tilde{f}$. It is a straightforward generalization of Fourier series theory on the circle that $\sigma_{j}$ converges uniformly to $f$ on $X_{T}$. So, in order to show $f \in R\left(X_{T}\right)$, we need only to show $\sigma_{j}$ 's in $R\left(X_{T}\right)$ for all $j$. Fix $z_{2}^{m} \mu$ in $S$,

$$
\begin{aligned}
\int \sigma_{j} z_{2}^{m} d \mu & \equiv \int_{T}\left(\frac{1}{2 \pi i} \int_{\Gamma_{z_{1}}} \sigma_{j}\left(z_{1}, z_{2}\right) z_{2}^{m} \frac{d z_{2}}{z_{2}}\right) d \nu\left(z_{1}\right) \\
& =\left\{\begin{array}{l}
0, j<|m|, \\
\frac{j-|m|+1}{j} \int_{T} f_{-m}\left(z_{1}\right)\left(1-z_{1} \bar{z}_{1}\right)^{m / 2} d \nu\left(z_{1}\right), j>|m| .
\end{array}\right.
\end{aligned}
$$

As $j \rightarrow \infty, \int \sigma_{j} z_{2}^{m} d \mu \rightarrow \int f z_{2}^{m} d \mu=0$ by hypothesis, while the right hand side approaches $\int_{T} f_{-m}\left(z_{1}\right)\left(1-z_{1} \bar{z}_{1}\right)^{m / 2} d \nu\left(z_{1}\right)$. So, we get $\int_{T} f_{-m}\left(z_{1}\right)\left(1-z_{1} \bar{z}_{1}\right)^{m / 2} d \nu\left(z_{1}\right)=$ 0 for all $\nu$ in $R(T)^{\perp}$. Therefore, $f_{-m}\left(z_{1}\right)\left(1-z_{1} \bar{z}_{1}\right)^{m / 2}=h_{-m}\left(z_{1}\right)$ for some $h_{-m}\left(z_{1}\right)$ in $R(T)$. And

$$
\begin{aligned}
\sigma_{j}\left(z_{1}, z_{2}\right) & =\frac{1}{j} \sum_{n=0}^{j} \sum_{k=-n}^{n} f_{k}\left(z_{1}\right) e^{i k \theta} \\
& =\frac{1}{j} \sum_{n=0}^{j} \sum_{k=-n}^{n} h_{k}\left(z_{1}\right)\left(1-z_{1} \bar{z}_{1}\right)^{k / 2} e^{i k \theta} \\
& =\frac{1}{j} \sum_{n=0}^{j} \sum_{k=-n}^{n} h_{k}\left(z_{1}\right) z_{2}^{k}
\end{aligned}
$$

is in $R\left(X_{T}\right)$. So is $f$.

We can now assert that the linear span of $S$ is weak-* dense in $R\left(X_{T}\right)^{\perp}$. For, if not, then there exists $g$ in $C\left(X_{T}\right)$ such that $g$ annihilates $S$ as well as its linear span, 
yet $\int g d \tau \neq 0$ for some $\tau \in R\left(X_{T}\right)^{\perp}$ which is not in the span of $S$. By (\#), $g$ is in $R\left(X_{T}\right)$. Hence $\int g d \tau=0$, a contradiction. So the linear span of $S$ is weak-* dense in $R\left(X_{T}\right)^{\perp}$. Q.E.D.

Proof of LeMMA.

$$
\begin{aligned}
\frac{\partial \phi_{0}}{\partial \bar{\zeta}_{1}} & =\frac{\partial}{\partial \bar{\zeta}_{1}}\left[\int_{-\pi}^{\pi} \tilde{\phi}\left(\zeta_{1}, t\right) \frac{d t}{2 \pi}\right] \\
& =\int_{-\pi}^{\pi}\left[\frac{\partial \phi}{\partial \bar{\zeta}_{1}}+\frac{\partial \phi}{\partial \zeta_{2}} \frac{-\zeta_{1}}{2\left(1-\zeta_{1} \bar{\zeta}_{1}\right)^{1 / 2}} e^{i t}+\frac{\partial \phi}{\partial \bar{\zeta}_{2}} \frac{-\zeta_{1}}{2\left(1-\zeta_{1} \bar{\zeta}_{1}\right)^{1 / 2}} e^{-i t}\right] \frac{d t}{2 \pi} .
\end{aligned}
$$

On the other hand,

$$
\frac{\partial \tilde{\phi}}{\partial t}=\frac{\partial \phi}{\partial \zeta_{2}} \frac{\partial \zeta_{2}}{\partial t}+\frac{\partial \phi}{\partial \bar{\zeta}_{2}} \frac{\partial \bar{\zeta}_{2}}{\partial t}=i \frac{\partial \phi}{\partial \zeta_{2}}\left(1-\zeta_{1} \bar{\zeta}_{1}\right)^{1 / 2} e^{i t}-i \frac{\partial \phi}{\partial \bar{\zeta}_{2}}\left(1-\zeta_{1} \bar{\zeta}_{1}\right)^{1 / 2} e^{-i t}
$$

So,

$$
\frac{\partial \phi}{\partial \zeta_{2}} e^{i t}=\frac{1}{i} \frac{\partial \tilde{\phi}}{\partial t} \frac{1}{\left(1-z_{1} \bar{z}_{1}\right)^{1 / 2}}+\frac{\partial \phi}{\partial \bar{\zeta}_{2}} e^{-i t}
$$

Substituting the above into (4), we get

$$
\begin{aligned}
\frac{\partial \phi_{0}}{\partial \bar{\zeta}_{1}} & =\int_{-\pi}^{\pi}\left(\frac{\partial \phi}{\partial \bar{\zeta}_{1}}+\frac{1}{2 i} \frac{-\zeta_{1}}{1-\zeta_{1} \bar{\zeta}_{1}} \frac{\partial \tilde{\phi}}{\partial t}+\frac{\partial \phi}{\partial \bar{\zeta}_{2}} \frac{-\zeta_{1}}{\left(1-\zeta_{1} \bar{\zeta}_{1}\right)^{1 / 2}} e^{-i t}\right) \frac{d t}{2 \pi} \\
& =\int_{-\pi}^{\pi}\left(\frac{\partial \phi}{\partial \bar{\zeta}_{1}}+\frac{\partial \phi}{\partial \bar{\zeta}_{2}} \frac{-\zeta_{1}}{\zeta_{2}}\right) \frac{d t}{2 \pi}=\left(\frac{\partial \phi}{\partial \bar{\zeta}_{1}}-\frac{\zeta_{1}}{\zeta_{2}} \frac{\partial \phi}{\partial \bar{\zeta}_{2}}\right)_{0} .
\end{aligned}
$$

The term $\int(\partial \tilde{\phi} / \partial t)(d t / 2 \pi)=0$, since, on $\Gamma_{\zeta_{1}}, \partial \tilde{\phi} / \partial t=\Sigma_{n=-\infty}^{\infty}($ in $) \phi_{n} e^{i n t}$ has no constant term. Q.E.D.

It is an immediate consequence of (\#) that we have

THEOREM 2. Let $\phi$ be a smooth function with $\partial \phi / \partial \bar{z}_{i} \equiv 0$ on $X_{T}, i=1,2$. Then $\phi \in R\left(X_{T}\right)$.

Proof. Since $\phi$ is annihilated by all elements of $S$ so by (\#) $\phi \in R\left(X_{T}\right)$. Q.E.D.

Next, we state a theorem about tame measures in general which is derived from the proof of a theorem due to Weinstock [2, Theorem 1.4].

TheOREM (Weinstock). Let $X$ be a compact subset of $\mathbf{C}^{n}$. If $\mu$ is a tame measure on $X$ and $\left\{U_{\alpha}\right\}_{1}^{N}$ is a finite open covering of $X$, then there exist $\mu_{\alpha}$ orthogonal measures for $R\left(X \cap \bar{U}_{\alpha}\right)$, where each $\mu_{\alpha}$ has its support contained in $X \cap U_{\alpha}$, and $\mu=\Sigma_{1}^{N} \mu_{\alpha}$. 
Proof. Let $\left\{\sigma_{i}\right\}_{i=1}^{n}$ be measures supported on $X$, such that $\mu=-\sum_{i=1}^{n} \partial \sigma_{i} / \partial \bar{z}_{i}$. Let $\left\{\phi_{\alpha}\right\}$ be a smooth partition of unity subordinate to $\left\{U_{\alpha}\right\}$ satisfying

(i) $0 \leqslant \phi_{\alpha} \leqslant 1$ and supp $\phi_{\alpha} \subset U_{\alpha}$,

(ii) $\Sigma_{1}^{N} \phi_{\alpha}=1$.

Then,

$$
\begin{aligned}
\mu & =-\sum_{i=1}^{n} \frac{\partial}{\partial \bar{z}_{i}}\left(\left\{\sum_{\alpha=1}^{N} \phi_{\alpha}\right\} \sigma_{i}\right) \\
& =-\sum_{i=1}^{n} \sum_{\alpha} \frac{\partial \phi_{\alpha}}{\partial \bar{z}_{i}} \sigma_{i}-\sum_{i=1}^{n} \sum_{\alpha} \phi_{\alpha} \frac{\partial \sigma_{i}}{\partial \bar{z}_{i}} \\
& =-\sum_{\alpha} \sum_{i} \frac{\partial \phi_{\alpha}}{\partial \bar{z}_{i}} \sigma_{i}-\sum_{\alpha} \phi_{\alpha} \sum_{i} \frac{\partial \sigma_{i}}{\partial \bar{z}_{i}} \\
& =-\sum_{\alpha}\left\{-\sum \frac{\partial \phi_{\alpha}}{\partial \bar{z}_{i}} \sigma_{i}+\phi_{\alpha} \mu\right\} \\
& =\sum \mu_{\alpha}, \quad \text { where } \mu_{\alpha}=-\sum_{i=1}^{n} \frac{\partial \phi_{\alpha}}{\partial \bar{z}_{i}} \sigma_{i}+\phi_{\alpha} \mu .
\end{aligned}
$$

To show that $\mu_{\alpha} \perp R\left(X \cap \bar{U}_{\alpha}\right)$, for any $g \in R_{0}\left(X \cap \bar{U}_{\alpha}\right)$,

$$
\begin{aligned}
\int g d \mu_{\alpha} & =-\sum_{i=1}^{n} \int g \frac{\partial \phi_{\alpha}}{\partial \bar{z}_{i}} d \sigma_{i}+\int g \phi_{\alpha} d \mu \\
& =-\sum_{i=1}^{n} \int g \frac{\partial \phi_{\alpha}}{\partial \bar{z}_{i}} d \sigma_{i}+\sum_{i=1}^{n} \int \frac{\partial\left(g \phi_{\alpha}\right)}{\partial \bar{z}_{i}} d \sigma_{i} \\
& =-\sum_{i=1}^{n} \int g \frac{\partial \phi_{\alpha}}{\partial \bar{z}_{i}} d \sigma_{i}+\sum_{i=1}^{n} \int g \frac{\partial \phi_{\alpha}}{\partial \bar{z}_{i}} d \sigma_{i} \text { since } \frac{\partial g}{\partial \bar{z}_{i}}=0 \forall i \\
& =0 .
\end{aligned}
$$

So $\mu_{\alpha}$ annihilates $R_{0}\left(X \cap \bar{U}_{\alpha}\right)$, hence will annihilate its closure $R\left(X \cap \bar{U}_{\alpha}\right)$. Since supp $\mu_{\alpha} \subset \operatorname{supp} \phi_{\alpha} \cap \operatorname{supp} \mu$; we have that supp $\mu_{\alpha} \subset X \cap U_{\alpha}$ and the theorem is proved. Q.E.D.

With the help of (\#) and the above theorem we can now assert that $R\left(X_{T}\right)$ is a local algebra.

THeOReM 3. Let $\left\{U_{\alpha}\right\}$ be a finite open covering of $X_{T}$. Let $f \in C\left(X_{T}\right)$ be such that the restriction of $f$ to $X_{T} \cap \bar{U}_{\alpha}$ is in $R\left(X_{T} \cap \bar{U}_{\alpha}\right)$ for all $\alpha$. Then $f$ is in $R\left(X_{T}\right)$.

Proof. Let $S$ be as in Theorem 1. For any $\mu \in S, \mu$ is tame by Theorem 1. It follows from the above theorem that there exist $\mu_{\alpha}$ 's such that supp $\mu_{\alpha} \subset X_{T} \cap U_{\alpha}$, $\mu_{\alpha} \perp R\left(X_{T} \cap \bar{U}_{\alpha}\right)$ and $\Sigma \mu_{\alpha}=\mu$. Hence, by hypothesis,

$$
\int_{X_{T}} f d \mu=\sum_{\alpha} \int_{X_{T} \cap \bar{U}_{\alpha}} f d \mu_{\alpha}=0
$$

$f$ is annihilated by $S$, and $f$ is then in $R\left(X_{T}\right)$ by (\#). Q.E.D. 
ACKNOWLEDGEMENT. This paper contains part of the results of the author's Ph.D. thesis written at Brown University under the direction of Professor John Wermer, to whom the author is deeply grateful.

\section{REFERENCES}

1. R. Basener, On rationally convex hulls, Trans. Amer. Math. Soc. 182 (1973), 353-381.

2. B. M. Weinstock, Approximation by holomorphic functions on certain product sets in $\mathbf{C}$, Pacific J. Math. 43 (1972), 811-822.

Department of Mathematics, Brown University, Providence, Rhode Island 02912

Current address: Institute of Mathematics, Academia Sinica, Taipei, Taiwan, Republic of China 